\title{
DETECTING KNOCK IN SPARK IGNITED ENGINES
}

\author{
Ylva Nilsson and Erik Frisk
}

\author{
Vehicular Systems, Dept. of Electrical Engineering \\ Linköping University, SE-581 83 Linköping, Sweden \\ Email: $\{y$ lvan, frisk\}eisy.liu.se
}

\begin{abstract}
In this paper we consider signal processing algorithms for detecting knock, i.e. auto-ignition of unburned fuel, in spark-ignited engines. To operate an engine with optimal efficiency, there is a need to operate close to the knock limit. This motivates the need for efficient knock detection algorithms that not only indicates knock, but also gives estimates on knock timing and size. The proposed detection algorithms are based on either incylinder pressure or ion current and both off-line and on-line methods are developed. The methods are tested on measured data, analysed, and compared with respect to detection performance, knock timing estimation accuracy, and robustness against model uncertainties. Copyright ${ }^{\circledR} 2005$ IFAC.
\end{abstract}

Keywords: knock, detection algorithms, engine, cylinder pressure, ion current, oscillations

\section{INTRODUCTION}

Due to economical and environmental concerns it is desirable to have an internal combustion engine with as high efficiency as possible. However, in many operating points, high efficiency operation increases the top temperature in the cylinder, and thereby the risk of auto-ignition (Stone, 1999). Auto-ignition of the unburned fuel in front of the flame front can be very harmful to the engine. Since the end gas is combusted very fast, it initiates a pressure wave - the so called knock. Severe knock can cause engine failure and even engine break down (Fitton and Nates, 1996).

Knock tendency is reduced by the engine control unit by retarding the ignition angle when a knock is detected. The ignition angle is then slowly moved back to its optimum, as long as no knock is discovered (Kiencke and Nielsen, 2000). Modest knock is not harmful to the engine. If the engine can be controlled towards a harmless knock intensity level instead of zero knock, efficiency can be increased in many cases. This implies that efficient knock detection methods can be a means to improve engine efficiency.

There are several different methods to detect knock (Kiencke and Nielsen, 2000). A common method is to mount a mechanical vibration sensor at the engine block. One can also measure the light intensity and colour in the combustion chamber. The two signals used in this paper are high pass filtered cylinder pressure and ion current.

\section{THE KNOCK SIGNATURE}

Ideally, the auto-ignition that initiates knock has the same effect on the cylinder pressure as hitting a drum has on the drumhead. An impulse, caused by the instantaneous combustion of an amount of fuel, is followed by damped oscillations as shown in Fig. 1. The oscillations are also visible in the ion current.

The frequency of the oscillation is determined by the geometry of the combustion chamber, where the fundamental oscillatory mode is a wave travelling from one side of the combustion chamber to the opposite side (Carstens-Behrens et al., 2002). Using a well tuned high pass filter, the oscillating knock signal can then be detected. Note that the characteristics and placement of the pressure sensor may make it more difficult to observe the fundamental mode than to observe the harmonics (Sawamoto et al., 1987). 

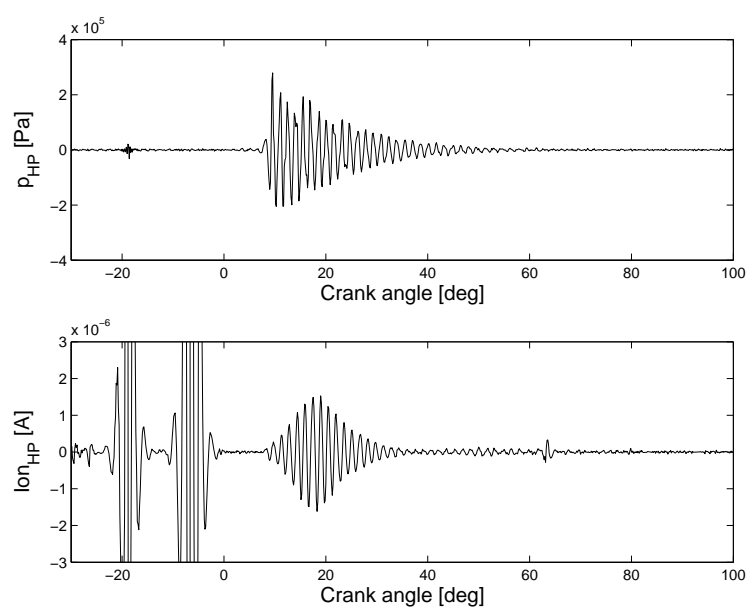

Fig. 1. Top: High pass filtered cylinder pressure. Bottom: High pass filtered ion current for the same cycle as in top figure.

The wave travels with the speed of sound $c$, that is

$$
c=\sqrt{\frac{\gamma R T}{M}}
$$

where $\gamma$ is the ratio between specific heats, $R$ the universal gas constant, $T$ cylinder gas temperature, and $M$ molar weight. The knock frequency can be calculated from the Bessel number (Blunsdon and Dent, 1994) and (1). For the engine used here, the frequency is approximately $8 \mathrm{kHz}$. The frequency changes noticeably during the oscillation (Härle and Böhme, 1987). For the pressure signal shown in Fig. 1, it changes from approximately $7920 \mathrm{~Hz}$ in the region $\theta \in\left[9^{\circ}, 28^{\circ}\right]$ to $7440 \mathrm{~Hz}$ in the region $\theta \in\left[35^{\circ}, 65^{\circ}\right]$.

\section{EXPERIMENTAL SET-UP}

Measurements were done on a SAAB Variable Compression Engine (Drangel and Reinmann, 2002). The engine is a highly boosted, 1.6 liter, 5 cylinder, spark ignited engine. The compression ratio can be changed between $r_{c}=8$ and 14 .

The tests were done in an engine test bench. Cylinder pressure and ion current were measured time-based at a sampling frequency of $82 \mathrm{kHz}$. Cylinder pressure was measured with an asymmetrically placed flushmounted piezo-electric sensor. Table 1 shows the investigated engine operating points. In each operating point, 99 consecutive cycles were measured.

\section{OFF-LINE METHOD (OFF)}

In the methods to follow, let $y$ denote HP filtered cylinder pressure or ion current. For the derivation of the off-line method, a signal model for the ideal knock signature in $y$ is needed. Let $\alpha=\left(t_{\text {knock }}, A, r\right)$ be a vector of the unknown parameters. An ideal knock signature, according to Section 2 can then be written
Table 1. Measured operating points (OP), where $p_{\text {im }}$ is inlet manifold pressure $[\mathrm{kPa}]$, $N$ engine speed [rpm], $r_{\mathrm{c}}$ compression ratio, and $\theta_{\text {ign }}$ the ignition angle [deg bTDC].

\begin{tabular}{rrrrr} 
OP & $p_{\text {im }}$ & $N$ & $r_{\mathrm{c}}$ & $\theta_{\text {ign }}$ \\
\hline 1 & 70 & 1200 & 9 & $12^{\circ}$ \\
2 & 70 & 1200 & 9 & $30^{\circ}$ \\
3 & 70 & 1200 & 14 & $12^{\circ}$ \\
4 & 70 & 1200 & 14 & $24^{\circ}$ \\
5 & 70 & 2000 & 14 & $28^{\circ}$ \\
6 & 70 & 3000 & 9 & $12^{\circ}$ \\
7 & 70 & 3000 & 14 & $12^{\circ}$ \\
8 & 100 & 1200 & 10 & $30^{\circ}$ \\
9 & 100 & 1200 & 14 & $4^{\circ}$ \\
10 & 100 & 2000 & 10 & $35^{\circ}$ \\
11 & 100 & 2000 & 14 & $9^{\circ}$ \\
12 & 100 & 3000 & 9 & $12^{\circ}$ \\
13 & 100 & 3000 & 10 & $35^{\circ}$ \\
14 & 100 & 3000 & 14 & $15^{\circ}$ \\
15 & 130 & 2000 & 9 & $12^{\circ}$ \\
16 & 130 & 2000 & 10 & $17^{\circ}$ \\
\hline
\end{tabular}

$$
\begin{aligned}
& \xi(t ; \alpha)=A r^{t-2-t_{\text {knock }}} \\
& \sin \left(\Omega\left(t-1-t_{\text {knock }}\right)\right) h\left(t-1-t_{\text {knock }}\right)
\end{aligned}
$$

where $\Omega$ is normalised frequency, $r$ the damping coefficient, $A$ the maximum amplitude of the knock, and $h(t)$ the step function. The model for the measured signal is

$$
y(t)=\xi(t ; \alpha)+e(t)
$$

where $e(t)$ is an independent sequence of $N\left(0, \sigma_{\text {noise }}^{2}\right)$ distributed, uncorrelated, stochastic variables.

A knock detection test based on $L$ data samples can in this setting be stated as an hypothesis test:

$$
\begin{aligned}
& H_{0}: \alpha \in \Theta_{0}=\left\{\left(t_{\text {knock }}, A, r\right): t_{\text {knock }}>L\right\} \\
& H_{1}: \alpha \in \Theta_{0}^{c}=\Theta_{1}
\end{aligned}
$$

In this statistical setting, it is natural to form a test quantity using the maximum likelihood ratio

$$
g=\max _{\alpha \in \Theta_{1}} \ln \frac{P_{\alpha}(y)}{P_{\alpha_{0}}(y)}
$$

For a given $\alpha, P_{\alpha}(y)$ is the multi-dimensional probability density function for the measured data. Using the ideal model (3) and the independence assumption we obtain a simplified expression for the test quantity as

$$
g=\max _{\alpha_{1} \in \Theta_{1}} \ln \prod_{i=1}^{L} \frac{P_{\alpha_{1}}(y(i))}{P_{\alpha_{0}}(y(i))}=\max _{\alpha_{1} \in \Theta_{1}} \sum_{i=n_{0}}^{L} s(i)
$$

where

$$
s(i)=\ln \frac{P_{\alpha_{1}}(y(i))}{P_{\alpha_{0}}(y(i))}=-\frac{(y(i)-\xi(i))^{2}}{2 \sigma_{\text {noise }}^{2}}+\frac{y(i)^{2}}{2 \sigma_{\text {noise }}^{2}}
$$

The test alarms when $g \cdot \sigma_{\text {noise }}^{2}$ is larger than a specified threshold and an estimate of the knock timing $t_{\text {knock }}$ is obtained by the maximising argument.

\section{ON-LINE METHODS}

Three on-line methods are investigated where the first is based on the idea that a knock increases the signal 
variance. The second method is a simplification of the off-line method and the last method uses the assumption that a knock changes the signal energy.

\subsection{On-line method I (ONI)}

A way to detect knock is to estimate the variance of the knock signal and supervise any changes. Assuming the expected signal value $\mu$ to be 0 , a change detection test can be written (Gustafsson, 2000):

$$
\begin{aligned}
& s(t)=y(t)^{2}-\hat{\sigma}_{y}^{2}(t-1) \\
& g(t)=\max (g(t-1)+s(t)-v, 0)
\end{aligned}
$$

and alarm if $g(t)>h$. As a rule of thumb, the drift $v$ should be chosen as one half of the expected change magnitude. The signal variance is estimated using:

$$
\hat{\sigma}_{y}^{2}(t)=\lambda \hat{\sigma}_{y}^{2}(t-1)+(1-\lambda) y^{2}(t) \quad \lambda \in[0,1]
$$

\subsection{On-line method II (ONII)}

A significant reduction in calculation operations compared to the off-line method in Section 4 is achieved by setting the amplitude of a knock oscillation to a constant and known value.

$$
\xi(t)= \begin{cases}\xi_{0}(t)=0, & t<t_{\text {knock }} \\ \xi_{1}(t)=A \sin \left(2 \pi f\left(t-t_{0}\right)\right), & t \geq t_{\text {knock }}\end{cases}
$$

The amplitude $A$ is set to $\beta \in[0,1]$ times the highest value of the HP filtered signal. To avoid the influence of outliers, the signal is filtered with a median filter. The time phasing $t_{0}$ is chosen in the range $\left[0, \frac{1}{f}\right]$, so that the highest correlation between $y$ and $\xi_{1}$ is achieved.

The hypotheses are the same as in (4), but with $A$ and $r=1$ fixed. The expected value of $y$ is $\mu_{0}(t)=0$ and $\mu_{1}(t)=\xi_{1}(t)$ respectively. Knock is detected using CUSUM and log-likelihood (Basseville and Nikiforov, 1993). Compute

$$
s(t)=\frac{\mu_{1}(t)-\mu_{0}}{\sigma_{\text {noise }}^{2}}\left(y(t)-\frac{\mu_{0}+\mu_{1}(t)}{2}\right), S_{1}^{j}=\sum_{i=1}^{j} s(i)
$$

Knock is detected if there exists a time $t_{\text {det }}$ such as

$$
t_{\mathrm{det}}=\min _{t}\left[\arg \left(S_{1}^{t}-\min _{1 \leq j \leq t} S_{1}^{j}\right)>h / \sigma_{\text {noise }}^{2}\right]
$$

Time of knock is set to the maximum $t<t_{\text {det }}$ that fulfilles $\left(S(t)-\min _{1 \leq j \leq t} S_{1}^{j}\right)=0$.

\subsection{On-line method III (ONIII)}

The signal energy over a limited time interval of $L$ samples, can be used to detect knock (Kiencke and Nielsen, 2000). The signal energy is

$$
E_{y}(t)=\int_{t}^{t+L} y^{2}(t) d t \approx \frac{1}{f_{s}} \sum_{k=k_{t}}^{k_{t}+L} y^{2}(k)=g(t)
$$

where $f_{s}$ is the sampling frequency. The signal is compared to a threshold $h \propto L$. Knock is detected if and when $g(t)>h$.

\subsection{Thresholds and design parameters}

The design parameters of the algorithms have to be chosen. In ONI, $\lambda$ is set to 0.95 , and the change magnitude is set to the 99 percentile of the noise distribution. In ONII, we need to set $\beta$. A too small $\beta$ has the disadvantage of making the test quantity sensitive to noise while a $\beta$ close to 1 is not representative since the amplitude of the oscillation decreases. The frequency of the model signal $\xi$ is set to the dominating frequency of the investigated crank angle region. The window length of ONIII is set to 10 samples, which is about one knock oscillation period.

To avoid the risk that a short, but strong, disturbance causes the tests to alarm, an additional requirement on the alarm is added. An alarm is issued only if the test quantity $g$ is higher than the threshold for at least 40 samples in succession, see Figure 2. The effective test quantity is

$$
g_{e}(t)=\min \{g(t), \ldots, g(t+40)\}
$$
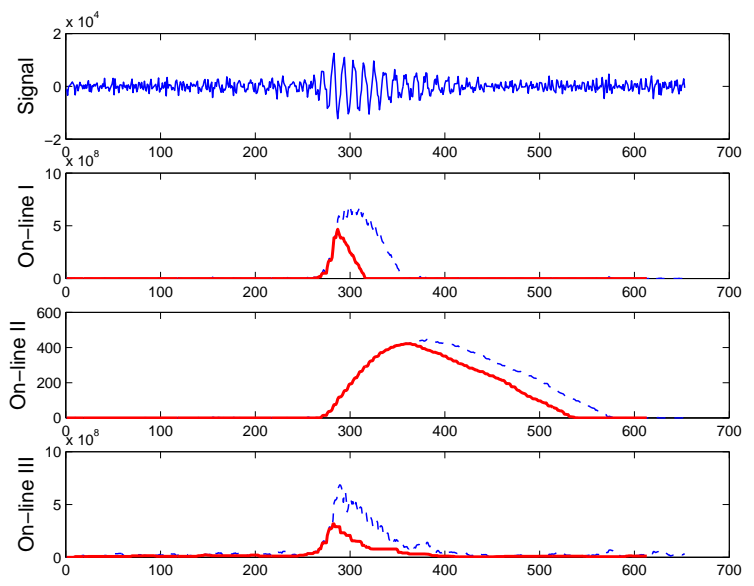

Fig. 2. The on-line test quantities during an engine knock. The thin line is the test $g$ and thick line the effective test $g_{e}$.

The thresholds of all methods are set to values such that knock is detected in exactly 3 cycles out of 99 in OP 3 , since a visual inspection gives that the number of knock occurrence is in the range 3-4.

\section{PRESSURE NOISE CHARACTERISTIC}

To determine whether the measured high pass filtered signal contains knock or not, knowledge about the noise characteristic is required. But, to know the characteristics during knocking condition, the noise has to be separated from the knock oscillations. However, a method for this separation is not readily available. 
A crank angle region that do not contain any knock is the compression phase. Therefore, an interesting question is if noise in this phase has similar characteristic as noise in the phase where knock appears, i.e. during combustion. The standard deviation and autocorrelation of the noise in the compression phase is compared to noise in the crank angle region $10^{\circ}-40^{\circ}$ after top dead center (TDC), for operating points with no or a low risk of knock. The HP filter used in the compression phase has a lower cut-off frequency than the one used in the combustion phase, since pressure waves travels slower in colder conditions according to (1). The standard deviation differs less than $10 \%$ between the phases. It is therefore assumed that the noise of the cylinder pressure in the compression phase is equivalent to the noise during combustion.

The standard deviation of the noise in the compression phase is shown in Table 2. It is of the same order of magnitude for all investigated operating points, but is slightly higher for $2000 \mathrm{rpm}$. A map of the standard deviations is thus used in the investigation to follow.

Table 2. Standard deviation for the noise in the compression phase.

\begin{tabular}{rlrl} 
OP & $\sigma_{\text {noise }}[\mathrm{kPa}]$ & OP & $\sigma_{\text {noise }}[\mathrm{kPa}]$ \\
\hline 1 & 1.61 & 9 & 1.61 \\
2 & 1.55 & 10 & 1.87 \\
3 & 1.60 & 11 & 1.81 \\
4 & 1.64 & 12 & 1.62 \\
5 & 1.62 & 13 & 1.61 \\
6 & 1.69 & 14 & 1.64 \\
7 & 1.67 & 15 & 2.24 \\
8 & 1.63 & 16 & 2.17 \\
\hline
\end{tabular}

Fig. 3 shows the noise distribution for one representative operating point and it is concluded that it is reasonable to assume the noise to be normal distributed. The auto-correlation curve is almost flat for engine speeds of 1000 and $3000 \mathrm{rpm}$, but at $2000 \mathrm{rpm}$ it has clear oscillations. All the same, the noise is forthwith considered white gaussian noise.
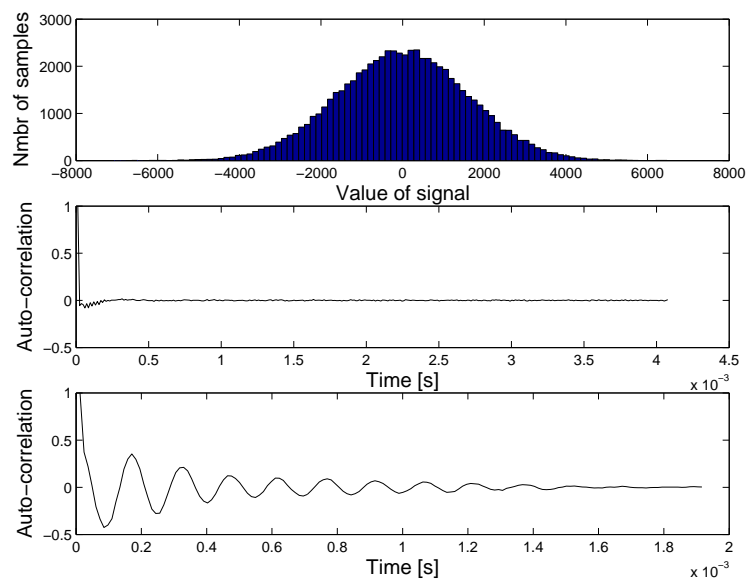

Fig. 3. Histogram for the pressure noise in OP 9. Auto correlation for OP 9 (center) and 16 (below).

\section{EVALUATION ON CYLINDER PRESSURE}

Now, the methods from the previous sections will be evaluated on pressure signals. A problem in the evaluation is that there are no non-disputable answers to the questions whether a cycle contains knock of not, and at what angle the knock is initiated. A light knock can be so modest that its oscillations can be mistaken for plain noise. The measured signal is HP filtered. If a causal filter is used a phase-shift is introduced, and if a zero-phase filter is chosen it creates small oscillations in the filtered signal before the time of auto-ignition. A problem when determining auto ignition angle is that it seems like the end gas is not always combusted at the same instant, which gives an indistinct growth of the oscillation amplitude.

Here, the cycles that contains knock and the autoignition angles $\theta_{\text {ref, } c i}$, is determined by visual inspection. Cycles where the knock signature is too unclear are not used in the investigation of standard deviation to follow. This is the case for many of the knocks in operating points at $3000 \mathrm{rpm}$.

\subsection{Knock angle}

A measure of knock angle estimation performance for each method is shown in Table 3. The estimation performance measure is the estimated standard deviation

$$
d=\sqrt{\frac{1}{n_{\text {cycles }}} \sum_{c i=1}^{n_{\text {cycles }}}\left(\theta_{\text {knock }, c i}-\theta_{\text {ref }, c i}\right)^{2}}
$$

where $\theta_{\mathrm{knock}, c i}$ is the detected auto-ignition angle for cycle $c i$, using one of the method in Section 4-5.

Table 3. Estimated knock angle standard deviation. Only operating points with more than 20 cycles with correctly detected knocks, are shown in the table.

\begin{tabular}{rllll} 
OP & OFF & ONI & ONII & ONIII \\
\hline 4 & $0.49^{\circ}$ & $0.43^{\circ}$ & $0.45^{\circ}$ & $0.17^{\circ}$ \\
5 & $0.67^{\circ}$ & $0.40^{\circ}$ & $1.23^{\circ}$ & $1.28^{\circ}$ \\
8 & $1.39^{\circ}$ & $0.34^{\circ}$ & $0.47^{\circ}$ & $0.21^{\circ}$ \\
9 & $0.76^{\circ}$ & $0.51^{\circ}$ & $0.55^{\circ}$ & $0.41^{\circ}$ \\
10 & $5.10^{\circ}$ & $0.92^{\circ}$ & $3.15^{\circ}$ & $1.74^{\circ}$ \\
11 & $0.66^{\circ}$ & $0.72^{\circ}$ & $1.43^{\circ}$ & $1.47^{\circ}$ \\
16 & $3.10^{\circ}$ & $0.72^{\circ}$ & $1.14^{\circ}$ & $1.65^{\circ}$ \\
\hline
\end{tabular}

Except for OP 10 and OP 16 the result is good for all methods, since $d<1.8^{\circ}$. ONI has the best performance with $d<1^{\circ}$ for all OP. Both the off-line method and ONII fails to detect several knocks in OP 10 , and the knock angle estimation is poor. The problem is caused by the model signal consisting of a single oscillatory frequency, while in many of the cycles of OP 10, the knock has not one dominating frequency but 2 or 3 . 


\subsection{Robustness}

To evaluate robustness properties of the algorithms, sensitivity towards changes in noise level, window length, and thresholds are investigated. Operating point 9 is used, since it contains many knocks in a wide range of intensities. Noise sensitivity is analysed by adding a vector with random numbers equally distributed as the measurement noise, but with higher variance. There are two possible situations, the algorithms are aware of the increased noise level or they are not. Algorithm performance in these two situations are shown in Fig. 4 and Fig. 5. It is seen that ONIII has problems with high noise levels. In Fig. 4 it is seen that the number of knocks detected by ONI decreases, and this is explained by that the expected change magnitude increases. But as can be seen in Fig. 5, not adjusting the change magnitude in ONI makes angle estimation unreliable.

The influence of varying the window length of the effective test value $g_{e}$ in (5) is shown in Fig. 6. The result of ONII on OP 9, is unaffected by changes in the window size in the investigated range. This can be explained by the flat appearance of test quantity ONII in Fig. 2. The results of the other two online methods changes with window size, but none of the methods have a deviation higher than $6 \%$ in the number of detected knocks, compared to window size of 40 samples. The changes in estimated knock angle deviations are less than $0.2^{\circ}$.

When the threshold is increased in Fig. 7, naturally the number of detected knocks decreases and vice versa. The rate of decrease is the same for all methods. The change in thresholds $h$ ranges from a factor $1 / 4$ to 4. Still the change in mean estimated knock angle deviation is $0.5^{\circ}$ at most.
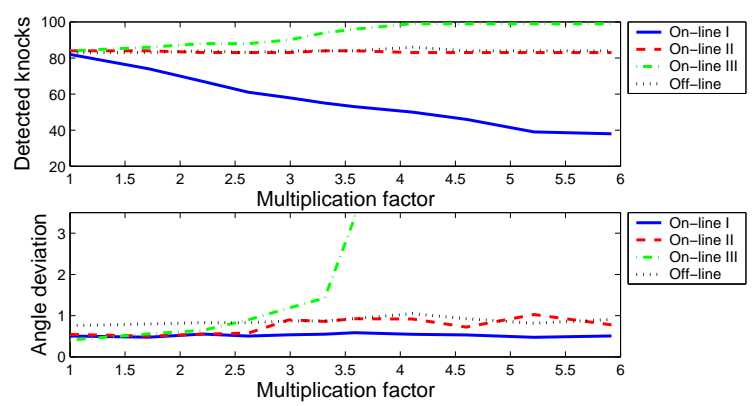

Fig. 4. Results of methods when noise is added to the cylinder pressure (OP 9). The design parameters are adjusted with the new noise level. Multiplication factor $k$ represents noise level $k \cdot \sigma_{\text {noise }}$.

\section{EVALUATION ON ION CURRENT}

The methods are also evaluated on ion currents. As before operating point 9 is used. The ion current contains some huge disturbances, that in some regions
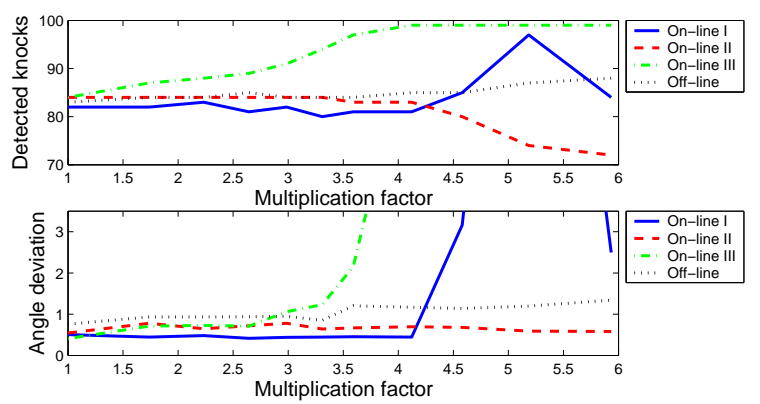

Fig. 5. Results of methods when noise is added to the cylinder pressure (OP 9), without adjusting the design parameters. Multiplication factor $k$ represents noise level $k \cdot \sigma_{\text {noise }}$.
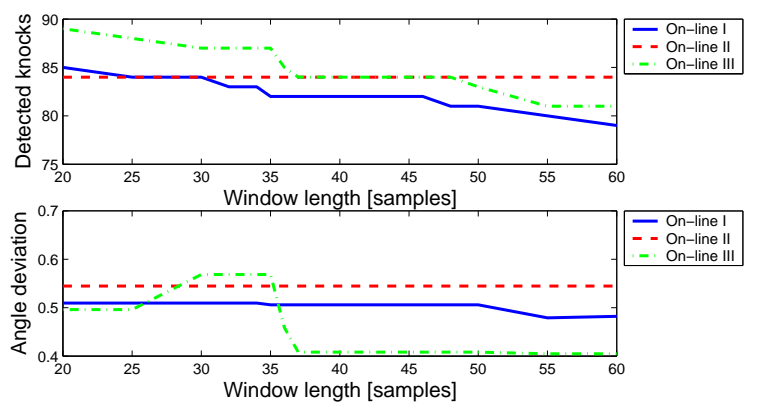

Fig. 6. Results of methods on OP 9 when the window size of the effective test (5) is changed.
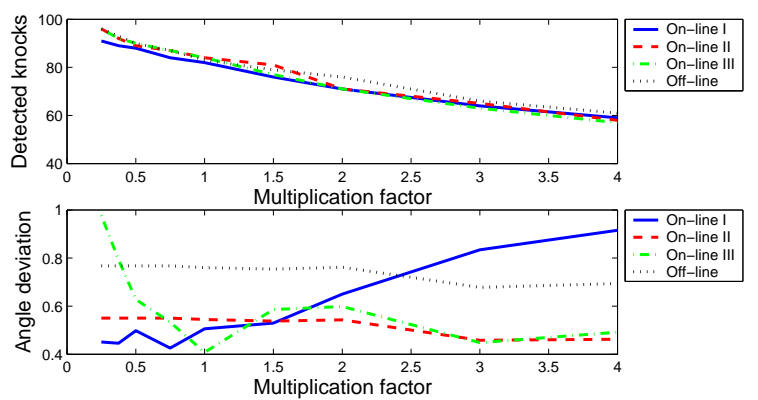

Fig. 7. Results of methods for different values of thresholds on OP 9. Multiplication factor $k$ represents the threshold $k \cdot h$.

completely drown the combustion information (see Fig. 1). These are due to the ignition functionality of the spark plug. The usable crank angle region is therefore shorter than for the cylinder pressure. The here measured ion current signal has a worse signal to noise ratio than the cylinder pressure. This means that it is harder to detect knock in the ion current than the cylinder pressure. Another difficulty is that the signal contains some heavy outliers, but this is effectively handled by the alarm requirement in (5).

The results of the knock angle detection on ion current are shown in Fig. 8. The knock angle standard deviations are in the range $1.1-1.3^{\circ}$.

About half as many knocks are detected in OP 9 based on ion current, compared to on cylinder pressure. An interesting question is if the cycles that caused the highest test values $g_{e}$ based on cylinder pressure, are 

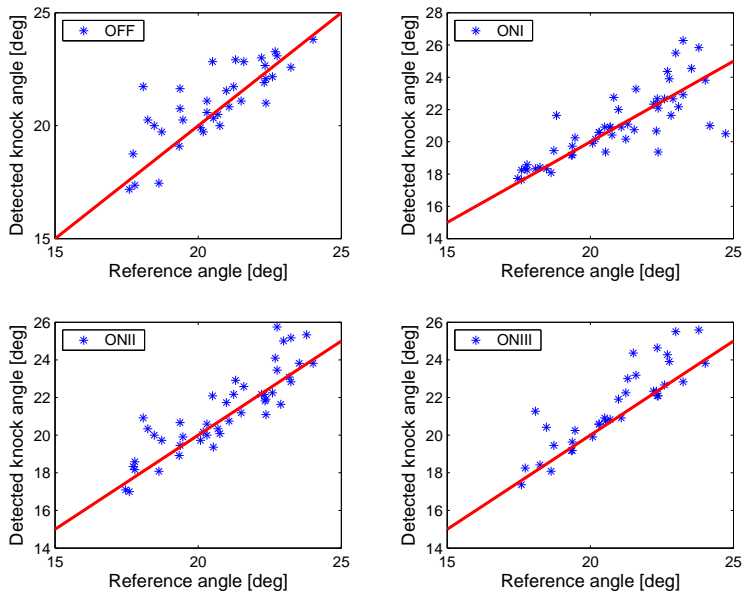

Fig. 8. Detected knock angle based on ion current. Idealy, the samples should be on the line $y=x$.

the ones that are detected in the ion current. Fig. 9 shows histograms of the test quantities, based on the pressure signal, for cycles where knock has been detected. The observation is that the cycles that is not detected based on the ion current may have both a low or a high maximum pressure test value.
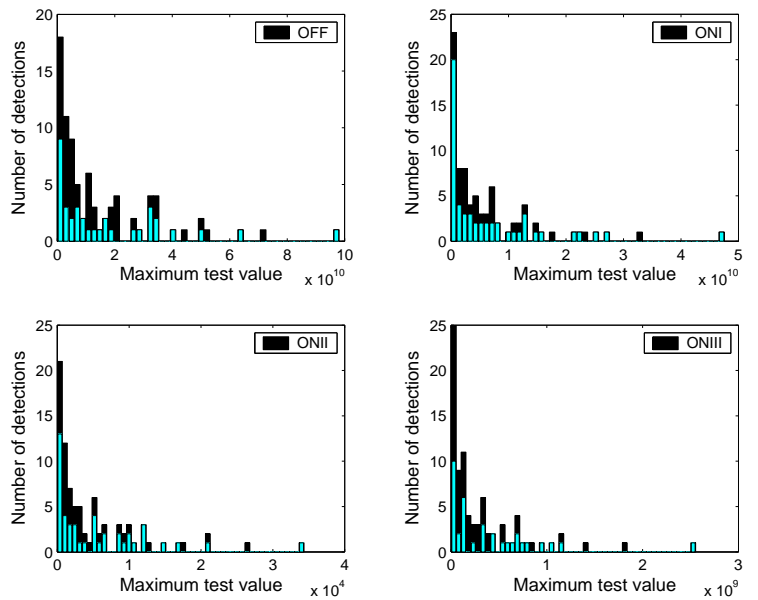

Fig. 9. Maximum effective test value based on cylinder pressure, for cycles with detected knock. The knock detection is based on pressure (black) and ion current (grey).

\section{CONCLUSIONS}

Four different methods for detecting knock and estimating auto-ignition angle from pressure and ion current signals have been investigated. When considering the results of the knock angle estimation based on the cylinder pressure, ONI has the smallest standard deviation between estimated and reference angle (less than $1^{\circ}$ ). With the exception of two operating points, the performance of the other methods are standard deviations up to $1.8^{\circ}$. The exceptions are OP 10 and 16 , where several of the measured cycles contain knock with a fundamental oscillatory tone that is not dominant. This is something that the off-line method and ONII have problems to handle.

Knock detection using the off-line method is the most computationally demanding. However, the performance of the method is no better than for the other methods. The reason for this is that the off-line method relies more heavily on the accuracy of the knock signal model (3) than the other methods and that the model have shown to be unreliable.

A weakness of ONI and ONIII is that they are sensitive to noise. The conclusion is thus that if there is a high signal to noise ratio, ONI is an appropriate choice of knock detection method. In a noisy environment ONII is a better choice.

The methods can be used to detect knock based on both cylinder pressure signals and ion currents.

Acknowledgement This work was financially supported by the Swedish Energy Agency.

\section{REFERENCES}

Basseville, M. and I.V. Nikiforov (1993). Detection of Abrupt Changes. PTR Prentice-Hall, Inc.

Blunsdon, C.A. and J.C. Dent (1994). The simulation of autoignition and knock in a spark ignition engine with disk geometry.

Carstens-Behrens, S., M. Urlaub, J.F. Böhme, J. Förster and F. Raichle (2002). Fem approximation of internal combustion chambers for knock investigations.

Drangel, H. and R. Reinmann (2002). The variable compression (SVC) and the combustion control (scc) - two ways to improve fuel economy and still comply with world-wide emission requirements. SAE Technical Paper 2002-02-0996.

Fitton, John and Roy Nates (1996). Knock erosion in spark-ignition engines.

Gustafsson, F. (2000). Adaptive Filtering and Change Detection. Wiley.

Härle, N. and J.F. Böhme (1987). Detection of knocking for spark ignition engines based on strucutral vibrations. Acoustics, Speech and Processing, IEEE International Conference 12, 1744-1747.

Kiencke, Uwe and Lars Nielsen (2000). Automotive Control Systems For Engine, Driveline, and Vehicle. Springer-Verlag.

Sawamoto, K., Y. Kawamura, T. Kita and K. Matsushita (1987). Individual cylinder knock control by detecting cylinder pressure. SAE paper No. 871911.

Stone, R. (1999). Introduction to Internal Combustion Engines. third ed.. SAE International. 